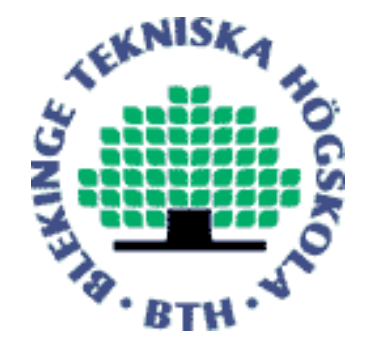

Copyright (C) 2012 IEEE.

Citation for the published paper:

On the ambiguity of Quality of Service and Quality of Experience requirements for eHealth services

Muhammad Ullah, Markus Fiedler, Katarzyna Wac

International Symposium on Medical Information and Communication Technology (ISMICT)

2012 La Jolla

This material is posted here with permission of the IEEE. Such permission of the IEEE does not in any way imply IEEE endorsement of any of BTH's products or services Internal or personal use of this material is permitted. However, permission to reprint/republish this material for advertising or promotional purposes or for creating new collective works for resale or redistribution must be obtained from the IEEE by sending a blank email message to pubs-permissions@iee.org.

By choosing to view this document, you agree to all provisions of the copyright laws protecting it. 


\section{On the Ambiguity of Quality of Service and Quality of Experience Requirements for eHealth Services}

\author{
Muhammad Ullah, Markus Fiedler \\ School of Computing \\ Blekinge Institute of Technology \\ Karlskrona, Sweden \\ \{muhammad.ullah, markus.fiedler\}@bth.se
}

\author{
Katarzyna Wac \\ Institute of Services Science, Quality of Life Group \\ University of Geneva \\ Geneva, Switzerland \\ Katarzyna.Wac@unige.ch
}

\begin{abstract}
The use of end-to-end communication systems as a medium of delivery for electronic healthcare (eHealth) services is considered to be uncertain, with respect to its reliability leading to hesitation in acceptance of such services. There exist different influential dimensions that pose stringent requirements on endto-end communication systems, influence user perception and might hinder user acceptance. The later is referred to as Quality of Experience (QoE), which among others depends on the Quality of Service $(Q o S)$ of the end-to-end communication system. $Q o E$ is considered as a key component determining user acceptance. This paper identifies and analyzes characteristics of a set of eHealth services and the influential dimensions resulting in different $Q o S$ requirements and potential impact of $Q o S$ on $Q o E$. It highlights the role of $Q o S$ and $Q o E$ for acceptance of these services. The issue of non-uniform views regarding $Q o S$ parameter specifications and related requirements, clinically acceptable thresholds and their qualitative representation in eHealth literature is reviewed and presented.
\end{abstract}

Keywords- QoE; QoS; eHealth; end-to-end communication system; user perception; user acceptance; expectations

\section{INTRODUCTION}

The application of emerging Information and Communication Technologies (ICT) in healthcare enable, in theory, provisioning of electronic healthcare (eHealth) services anytime and anywhere, eliminating the time and space barriers, by allowing remote access to patient information, laboratory results, pharmaceutical information, insurance information, medical resources - enabling the delivery of various type of date including audio, video, image and signal data, thereby improving the quality of patient care. However, due to the heterogeneity of end-to-end communication infrastructures, the ever-growing application and service domains of modern eHealth, the unpredictable context of use, the environmental setting and human factors, there exist many concerns with respect to eHealth acceptability. These concerns include the Quality of Service (QoS) provisioning (availability, data delivery latency, reliability) and mobility support [1, 2], privacy and security of information [3], cost, and user perceived quality in particular [4], that influence user acceptance of the service [5]. User acceptance is considered as function of user perception of the service quality, and the most important factor for any healthcare service implementation. As Broens et al. [6] present, the most important determinants for successful telemedicine implementation were reported as user acceptance $(37 \%)$ and technology deployment factors $(29 \%)$, leaving behind the organization (13\%), policy and legislation $(11 \%)$ and financing factors $(10 \%)$.

User perception is referred to as Quality of Experience $(Q o E)$, and depends on $Q o S$ provided by the underlying communication systems It (used interchangeably to) represents user satisfaction level of the service and result in his/her experience of the delivered service quality [5]. The interdependency of $Q o E$ and $Q o S$ has been reported as "two distinct constructs and related through a causal chain of environmental influences (QoS) that impact cognitive perception and behavioral consequences (QoE) of a user" [7]. The quantitative relationship between $Q o S$ and $Q o E$ for streaming and web surfing application has been presented in [8], and it was experimentally proven that an exponential relationship exist between $Q o S$ and $Q o E$. The practical use of healthcare technologies, and particularly "best effort" $Q o S$ level communication systems supporting the eHealth services, result in an uncertain level of service reliability, that in turn may lead to hesitation in acceptance [5] of such services by their end-users, i.e., healthcare professionals and patients. Practically, it is expected that all forms of eHealth should adhere to the same standards of safety, quality and usability, as that in a traditional healthcare, and the goal should be user satisfaction and system acceptance [9].

To deal with user satisfaction and acceptance, the notions of "expected" and "perceived" quality are identified. Where, $Q o E$ perceived by user should be equal or higher than $Q o S$ expected in order to satisfy the user [10]. To overcome the above-mentioned hindrance and to facilitate user acceptance, user perception of the delivered services needs to be identified and eventually improved. According [4, 11], "if the system cannot be trusted to guarantee a threshold level of quality, it will remain underutilized, be bypassed, or used as a measure of last resort". Authors argued that the strong influence of quality perceptions on one's inclination to avail health services is reported beyond dispute [4]. Author further argued that the "perceptions of poor quality of health care may, in fact, dissuade patients from using the available services because health concerns are among the most salient of human concerns" [4]. Therefore, to understand the user perception, the clinically acceptable thresholds of $Q o S$ parameters for the underlying communication systems need to be identified and their corresponding user perceptions need to be verified.

Literature shows that, on one hand, the impact of poor $Q o E$ and low $Q o S$ can result not only in a poor quality of healthcare service. For instance, data loss may lead to false judgments in diagnosis [12], data latency may affect the accuracy and completion time of surgical task [13] [14]. Similarly, delay in remote surgery may significantly degrade remote surgeon's task performance [15] and can results in his/her overall poor performance [16], in turn affecting his/her confidence level. On the other hand, the literature does not give a uniform view regarding the $Q o S$ parameters and their acceptable thresholds, and in turn, the causes of low $Q o E$ with respect to $Q o S$ cannot be pointed out clearly in operational eHealth systems.

The related work in this context is mainly focused on QoS provisioning and management. An overview of the eHealth technologies and wireless telemedicine systems are presented in [17]. Also a survey on QoS provisioning in wireless eHealth, handoff schemes for QoS support in wireless networks, the priority schemes for eHealth services and techniques/methods for IEEE 802.11 to guarantee QoS is also provided. A detailed analysis of QoS provision in eHealth services has been presented in [18], and it has been shown that eHealth services have diversified demands where the requirement of each 
application type vary with respect to context of use, type of service and the level of urgency. Authors in [19] proposed a methodology for the technical evaluation of QoS traffic requirement for telemedicine application, taking in to account application requirements, network topologies, and the evaluation of services using an automated tool. However, the identification of clinically acceptable QoS threshold and their impact on user QoE still need to be verified. In this context, this paper aims to identify and analyze characteristics of eHealth services and the influential dimensions contributing to their QoS requirements. It attempts to highlight the potential impact of QoS on QoE and their role in acceptance of these services. It also presents the issue of a non-uniform view regarding QoS parameter's specifications, clinically acceptable thresholds and their qualitative representation in eHealth literature. These issues do not help the potential service developers to derive the requirements posed by these services on the end-to-end communication system, adding more to the uncertainty of user acceptance.

The remainder of the paper is organized as follows. In Section 2, an overview of the influential dimensions on eHealth services and relation with $Q o S$ and $Q o E$ is presented. Section 3 presents two case studies about disagreements among researchers regarding $Q o S$ parameter's thresholds, and their qualitative representations. In section 4, we discuss the issues relating the user perception resulting from these disagreements. Conclusion and Outlook is provided in Section 5.

\section{INFLUENTIAL DimENSIONS OF EHEALTH}

eHealth systems rely on distributed service infrastructure and the underlying end-to-end communication system for service delivery. This communication system is provided by multiple network segments, as presented in Fig. 1. eHealth can deliver different services to patients and healthcare practitioners, including telemedicine services [19], monitoring services rely on wearable and portable systems, access to electronic health record (EHR), sharing of information and efficient resource utilization are few to mention. The eHealth service domain varies with respect to application area, application purpose, content type and context of use, reported as influential dimensions [20]. These dimensions define the QoS requirements from end-to-end communication system and contribute to the user perception. The user's state and end-toend system are dimensions influencing user perception besides the content and context of use (Fig. 1), as reported in [21].

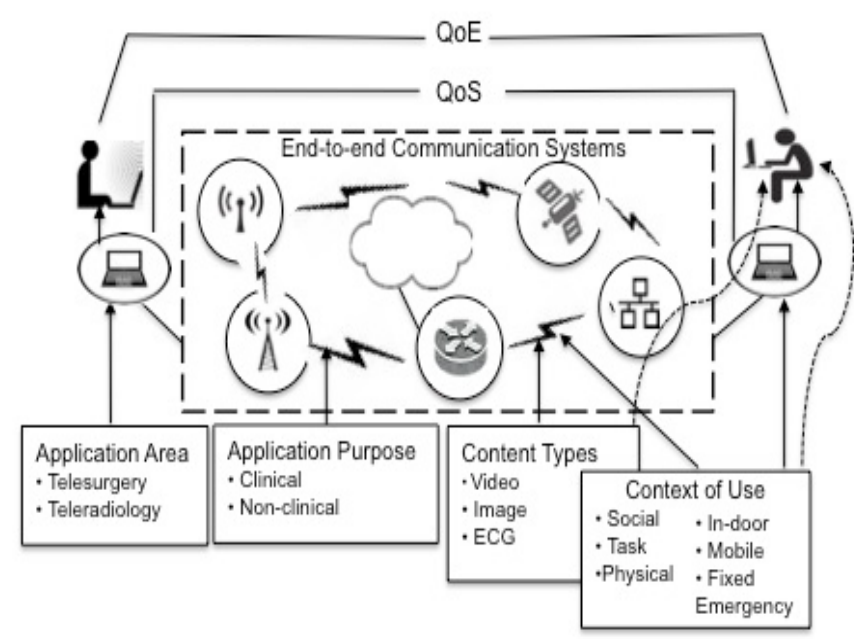

Figure 1. eHealth influential dimensions and its QoE

The application area in remote healthcare settings would initially define the required set-up to replace face-to-face interaction between distant users. Application purpose defines the type of interest i.e., professional, clinical interest, or nonclinical, educational interest on the one hand, it differentiates the level of requirements for the quality of the delivered services. For example, clinical applications will have more stringent requirements than their non-clinical counterparts.

eHealth applications can have different content types e.g., video, audio, image, access to EHR and or devices capturing vital signs (e.g., ECG). They have different service quality $(Q o S)$ requirements posed on the end-to-end communication system [2]. User perception can vary with respect to content types and their delivered quality level. For example, watching video trailers vs. videoconferencing will result in different user perception based on their interest level. But the impact of delivered quality level also influence user perception and its degradation might lead to service rejection [5].

eHealth applications can be delivered in different user context e.g., hospital care, primary care, emergency care. The requirements from end-to-end communication system vary with respect to the context of use e.g., real-time vs. non realtime or emergency vs. non-emergency situations [2]. The video transmission from the patient side to the remote surgeon in telesurgery will have a higher level of stringency in requirements than video transmission during routine consultation. Moreover, environment, location as context, e.g., in-door or in a moving ambulance, will affect the interaction with system results in different user perception and also pose different $Q o S$ requirements depending on which connectivity the user is using.

\section{EXAMPLE CASES OF DisAGREEMENTS IN THE LITERATURE}

In the following sections we give concrete examples of non-uniform view regarding $Q o S$ parameter specifications and requirements, clinically acceptable thresholds and their qualitative representation in the eHealth literature. 


\section{A. Telesurgery Service and Its Requirements}

The combination of computer-assisted telemanipulators, surgery and telecommunication resulted in robotic-assisted surgery called telesurgery or remote surgery. Telesurgery enable surgeons to perform surgical operations on distant patients, reducing the space and time barriers, i.e., a surgeon interacts with a robotic interface in one location, conveying action to the surgical robot at the patient's place. Surgical tasks are directly performed by the robotic system controlled by the surgeon at the remote site. The surgical tasks include remote diagnosis, treatment, evaluation; consultation for expert opinion; collaboration and telepresence of different surgeons for supervision and visualization of the operating field. All these surgical tasks are based on transmission of content types such as video, audio, image and vital signs and patient information and robotic control signals between patients and surgeons using end-to-end communication system.

Studies showed that in remote surgeries relying upon long communication links, time delay would be a major constraint resulting in poor user perception. Given the distribution of service users, the Round Trip Time (RTT) is considered as a QoS parameter of importance and it is defined as "the sum of control latency and visual discrepancy, i.e., the time between when a remote surgeon moves a controller and when such translated movement is visually appreciated at the remote location" [16]. The following paragraphs illustrate the example studies on the impact of RTT on $Q o E$ and surgeon's surgical skills, task completion time and accuracy.

In the study by Hanly et al. [16], the acceptable RTT threshold of $500 \mathrm{~ms}$ was reported, while authors argued that telesurgery could be possible even with $1000 \mathrm{~ms}$ delay (unpublished data), if some compensatory mechanisms, such as training of surgeon, slowing surgeon hand's movement, creating a virtual instrument in surgeon minds, or other techniques, are employed. Similarly, studies by Rosen et al. [13], Marescaux et al. [22], Anvari et al. [23], Perez et al. [15], and Rayman et al. [16], reported acceptable threshold of RTT as $<500,330,280,<300$ and $600 \mathrm{~ms}$, respectively, shown in Fig. 2. They depend on the type of the end-to-end communication systems (e.g., wired \& wireless, ATM, IP/VPN with $Q o S$ ) and different surgical operations (e.g., block transfer, gall-bladder removal, laparoscopic surgery, fundoplications, fixing nuts and passing needle from holes, object grasping and precise placement, and Internal Mammary Artery (IMA) dissection.

The RTT thresholds were reported in studies as relating to the user $Q o E$, defined as noticeable RTT and maximum tolerable RTT. Noticeable RTT was considered influencing surgeon perception of delivered quality, by being apparent on the remote surgeon screen, but was still acceptable to complete the surgical task. The maximum tolerable RTT was the threshold that affects the accuracy of the task, surgeon hand skills [16] and task completion time [16]. It is also noticed that level of difficulties was different with respect to type of task, even for the same amount of delay [15]. The variations in noticeable RTT (perceptual thresholds) and maximum tolerable RTT thresholds were reported in different studies as in Fig. 2.
On one side, the impact of poor $Q o S$ compromises reliability of the delivered service and influences the user perception. On other side, the variations reported in literature do not help in standardizing a threshold for particular $Q o S$ parameter. In practice, each study has a different setup, but the end users in telesurgery are not concerned with technical specification and complexity of the underlying end-to-end communication system for the intended intervention. Rather, they consider their expected and perceived quality during surgical operations.

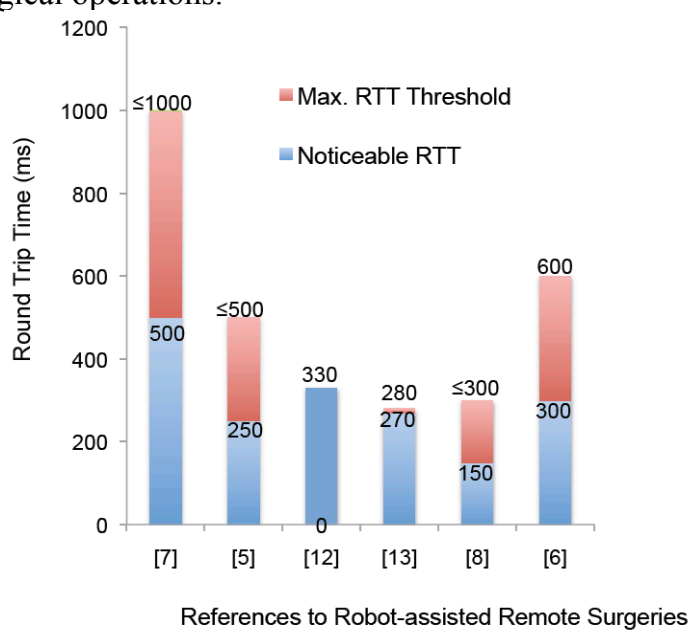

Figure 2. Noticeable and maximum tolerable RTT thresholds in telesurgery.

The non-uniform view represents the divergence of literature on $Q o S-Q o E$ requirement in telesurgery. The issue of disagreement on $Q o S-Q o E$ requirements leads to uncertainty when trying to figure out the actual causes of poor user perception, considering the quality delivered by the end-to-end communication system. Furthermore, the improvement to achieve the required level of $Q o S$ and facilitate the improvement of $Q o E$ is only possible when the requirements are identified.

\section{B. Qualitative Requirements}

There exists research postulating qualitative determination of thresholds for the $Q o S$ parameters. For example, a real-time mobile teletrauma system for non-emergency real-time prehospital care presented by Chu et al. [24] was implemented in a lab environment. They have shown the simultaneous transmission of video, images and ECG signals between patient and a physician. The authors found that different content types (video, images, ECG) required different priorities and level of sensitivity towards $Q o S$ parameters and suggested corresponding thresholds ("yes" - means allowed) (Table I).

TABLE I. QOS REQUIREMENTS IN TELE-TRAUMA SYSTEM

\begin{tabular}{|l|c|c|c|c|}
\hline $\begin{array}{c}\text { Service } \\
\text { Type }\end{array}$ & $\begin{array}{c}\text { End-to- } \\
\text { End } \\
\text { Delay }\end{array}$ & $\begin{array}{c}\text { Packet } \\
\text { Loss } \\
\text { Ratio }\end{array}$ & $\begin{array}{c}\text { Trans- } \\
\text { mission } \\
\text { Error }\end{array}$ & Video Resolution \\
\hline Video & No & Yes & & $320 \times 240 / 160 \times 120$ \\
\hline Image & Yes & No & & \\
\hline ECG & & No & No & \\
\hline
\end{tabular}


A wireless telemedicine system for remote patient care during a disaster has been proposed and demonstrated by $\mathrm{Chu}$ et al. [25]. This system allows simultaneous transmission of video, sensor information, images, and text base patient information. They demonstrated the remote control of information from various disaster centers between remote patients and a control center, serving a group of hospitals units of the disaster area. Data integrity was considered as a performance metric and transmission methods for different content types were recommended (Table II).

TABLE II. QOS REQUIREMENTS IN EMERGENCY TELEMEDICNE

\begin{tabular}{|l|c|c|}
\hline \multicolumn{1}{|c|}{ Service Type } & $\begin{array}{c}\text { Transmission } \\
\text { Method }\end{array}$ & Data Integrity \\
\hline ECG & TCP & High \\
\hline Medical Images & TCP & High \\
\hline Real Time Video & UDP & Low \\
\hline
\end{tabular}

Another view on qualitative thresholds for different $Q o S$ parameters in emergency healthcare applications has been presented by Vouyioukas, et al. [2]. The authors also categorized applications in real-time and near real-time and suggested $Q o S$ requirements in terms of throughput, jitter and delay for different medical emergency conducts including teleconsultation, telemonitoring, tele-education, telediagnosis and remote access to EHR (Table III).

TABLE III. QOS REQUIREMENTS IN EMERGENCY TELEMEDICINE

\begin{tabular}{|l|c|c|c|}
\hline \multicolumn{1}{|c|}{ Service Type } & $\begin{array}{c}\text { Required } \\
\text { Throughput }\end{array}$ & $\begin{array}{c}\text { Small } \\
\text { Delay }\end{array}$ & $\begin{array}{c}\text { Small } \\
\text { Jitter }\end{array}$ \\
\hline Teleconsultation & High & Yes & Yes \\
\hline Telediagnosis & High & Yes & No \\
\hline Telemonitoring & Low & Yes & No \\
\hline Tele-education VoD & High & No & No \\
\hline Access to HER & Low/High & No & No \\
\hline
\end{tabular}

Summarizing, the lack of a unified view on qualitative requirements of $Q o S-Q o E$ can be seen in the literature. On the one side, it does not contribute to understanding the cause of poor user perception, neither it facilitates derivation of quantitative bounds of $Q o S$ to assure the user perception.

\section{DISCUSSION}

This paper demonstrated some examples a non-uniform view on $Q o S$ requirements for eHealth services. For example, the RTT is considered as an important $Q o S$ requirement for telesurgery, yet the literature does not help in specifying any standard of a minimum required RTT threshold for the newly deployed services. Moreover, the qualitative representation of $Q o S$ requirements and their thresholds for diverse, authorspecific parameters cannot be correlated to any quantitative threshold as a standard. Also, these thresholds are defined with different scales such as "low", "medium" and "high", which cannot directly related to user $Q o E$ level of the delivered service. It is also obvious from the literature that user involvement and proper methodology to identify user subjective rating of delivered services quality are not employed. A measurement of the user $Q o E$ for the delivered service based on the established quality scale and identification of acceptable $Q o S$ thresholds, would be an essential part towards the solution.

In eHealth, service or application requirements can be very different depending on different dimensions (c.f. Section II). There exist various performance challenges (e.g., delay in long distance communication links) considering the influential dimensions and the service-ability of end-to-end communication systems. The impact of poor $Q o S$ on user perception is obvious from the literature. Moreover, in some services we require the joint transmission of different content types and they have different criticality nature (c.f. Section IIIB). Therefore, all influential dimensions need to be considered to fulfill the service requirements. To this end, ideally there would be some classification of service requirements based on the application area, purpose of conduct, context of use and most importantly the content type used in that service. To improve overall user perception, identification of required level of $Q o S$ and selecting an appropriate end-to-end communication system for the intended service shall be a starting point. Also, the best alignment of technology and healthcare applications is only possible when the clinical requirements for desired application are identified and evaluated rigorously. The key would be to deliver content according to the level of quality required to satisfy end-users and ensure their acceptability.

\section{CONCLUSION AND OUTLOOK}

The different forms of eHealth systems are progressively showing their advantages over traditional healthcare, but acceptance of such systems is still a challenging issue. To improve user acceptance of eHealth services, user perception represented by user experience level $(Q o E)$ needs to be improved. Although, provision of service quality $(Q o S)$ in eHealth applications has been under investigation for long time, but the lack of thresholds for $Q o S$ parameters is evident from literature. This non-uniformity regarding the $Q o S$ parameter thresholds does not contribute to quality assessment of the impact of $Q o S$ on the end-user $Q o E$. Therefore, in order to improve user perception of the delivered eHealth services and ensure user acceptance, a systematic classification of healthcare services considering all influential dimensions, their corresponding $Q o S$ and $Q o E$ parameters and their clinically acceptable thresholds should be identified and subsequently evaluated. Moreover, an appropriate methodology that incorporates the suggested guidelines for conducting $Q o E$ studies in eHealth, user involvement and other dimensions of $Q o E$, need to be considered and their relation with $Q o S$ should be identified. To sum-up, our future work embraces a detailed systematic literature review [26] to explore $Q o E$ dimensions in existing eHealth research and practices.

\section{ACKNOWLEDGEMENT}

This work is partially supported by the Swedish Knowledge Foundation (KKS) project 'Quality of Experience based Cross-Layer Design of Mobile Streaming on Demand' 
(QoEMoS) (Dnr 2008/0520), European AAL Projects MyGuardian (2011-4), WayFiS (2009-3-129) and TraiNutri (2010-2-014).

\section{REFERENCES}

[1] D.D.Vergados. Simulation and Modelling Bandwidth Control in Wireless Healthcare Information Systems. SIMULATION 2007. 2007.

[2] D. Vouyioukas, I. Maglogiannis, and D. Komnakos, "Emergency mHealth Services through High-Speed 3G Systems: Simulation and Performance Evaluation," Simulation, vol. 83, no. 4, pp. 329-345, 2007.

[3] A. Zvikhachevskaya, G. Markarian, and L. Mihaylova, "Quality of Service consideration for the wireless telemedicine and eHealth services," in Proceedings of the IEEE Wireless Communications and Networking Conference (WCNC '09), pp. 1-6, April 2009.

[4] Andaleeb, S.S., (2001) Service quality perceptions and patient satisfaction: a study of hospitals in a developing country. Social Science \& Medicine, 52(9), 1359-1370.

[5] R. Stankiewicz, P. Cholda, and A. Jajszczyk, "QoX: what is it really?" IEEE Communications Magazine, vol. 49, no. 4, pp.148-158, 2011.

[6] T. H. Broens, et al., "Determinants of successful telemedicine implementations: a literature study," Journal of Telemedicine and Telecare, vol. 13, no.6, pp. 303-309, 2007.

[7] W. Wu et al. "Quality of experience in distributed interactive multimedia environments: Toward a theoretical framework", In Proc. ACM MM'09, pages 481-490, 2009.

[8] M. Fiedler, T. Hoßfeld, and P. Tran-Gia, "A generic quantitative relationship between Quality of Experience and Quality of Service," IEEE Network, Special Issue on Improving Quality of Experience for Network Services, 2010.

[9] European Commission, "Together for Health: A Strategic Approach for the EU 2008-2013," White paper, IP/07/1571, 23 October 2007.

[10] E. Babulak, "Quality of service provision assessment in the healthcare information and telecommunications infrastructures," International journal of medical informatics, vol. 75, no. 3-4, pp. 246-252, 2006

[11] Dagger, T.S. \& Sweeney, C. J. (2006) The effect of service evaluations on behavioral intentions and quality of life, Journal of Service Research, 9(1), 3-18

[12] F. Courreges, P. Vieyres, R. S. Istepanian, P. Arbeille, and C. Bru, "Clinical trials and evaluation of a mobile, robotic tele-ultrasound system," J Telemed Telecare, vol. 11, Suppl 1, pp.46-49, 2005.

[13] M. H. J. Lum, et al., "Teleoperation in surgical robotics--network latency effects on surgical performance," IEEE EMBC, Minneapolis, MN, USA, 2009.

[14] R. Rayman, et al., " Effects of latency on telesurgery: an experimental study," in Proc. 8th Int. Conf. MICCI, Palm Springs, CA, 2005.

[15] E. J. Hanly, et al., "Dynamics and organizations of telesurgery. European Surgery," European Surgery, vol. 37, no.5, pp. 274-278, 2005.

[16] M. Perez, et al., "Paradigms and experimental set-up for the determination of the acceptable delay in telesurgery," Conference proceedings, IEEE EMBC, Lyon, France, 2007.

[17] I. Martínez, J.Salvador, J. Fernández, J. García. Traffic requirements evaluation for a telemedicine network. International Congress on Computational Bioengineering, España, 2, 2003

[18] L. Skorin-Kapov and M. Matijasevic, "Analysis of QoS requirements for e-Health services and mapping to evolved packet system QoS classes", International Journal of Telemedicine and Applications, Volume 2010 (2010), Article ID 628086, 18 pages.

[19] Bratan T and Clarke M. Towards the Design of a Generic Systems Architecture for Remote Patient Monitoring. IEEE Engineering in Medicine and Biology 27th Annual International Conference; Shanghai, China; 2005. p. 106-109.

[20] B. Tulu, S. Chatterjee, and M. Maheshwari, "Telemedicine taxonomy: a classification tool," Telemedicine journal and eHealth, vol. 13, no. 3, pp. 349-358, 2007

[21] R. Schatz, P. Reichl, "Quality of Experience-More Than Just Another Buzzword?," EuroView2011 Workshop, Aug 1-2, Wurzburg, Germany.
[22] J. Marescaux, et al., "Transatlantic Robot-Assisted Telesurgery", Nature, vol. 413, no. 6854, pp. 379-380, 2001.

[23] M. Anvari, C. McKinley, and H. Stein, "Establishment of the World's First Telerobotic Remote Surgical Service: For Provision of Advanced Laparoscopic Surgery in a Rural Community, "Annals of Surgery, vol. 241, no. 3, pp. 460-464, 2005.

[24] Y. Chu and A. Ganz, "A Mobile Teletrauma System using 3G Networks," IEEE Trans. Inform. Tech. Biomed.,vol. 8, no. 4, pp. 456562,2004 ,

[25] Y. Chu, A. Ganz, "WISTA: A Wireless Telemedicine System for Disaster Patient Care", Journal of Mobile Networks and Applications, vol. 12, no.2-3, pp. 201-214, 2007.

[26] B.A. Kitchenham, Guidelines for performing Systematic Literature Reviews in Software Engineering Version 2.3, Keele University and University of Durham, EBSE Technical Report, 2007. 